\title{
SRPH
}

\section{Marine Algae as a New Source of Bioactive Compositionsin}

\author{
Azin Ghafarizadeh ${ }^{1}$ and Azadeh Niroomand ${ }^{2}$
}

ALKHAS, 2021;3(4): 1-5

\author{
${ }^{1}$ Department of Biology, Faculty of \\ Science, Lorestan University, \\ Khoramabad, Iran. \\ ${ }^{2}$ Department of Biology, Payame \\ Noor University, PO BOX 19395- \\ 3697 Tehran, Iran.
}

\section{*Corresponding Author: \\ $>$ a_niroomand@pnu.ac.ir}

Received: 15 September, 2021

Accepted: 30 November, 2021

Published: 30 December, 2021

\begin{abstract}
Marine algae are ecologically important and have been used as food and medicines for centuries. Numerous novel compounds have been found from seaweed with interesting pharmaceutical activities. Therefore, marine algae are believed to be a potential source to provide not only novel biologically active substances for the development of pharmaceuticals. Several bioactive metabolites produced by marine algae have been discovered by screening programs. Many of these chemical compounds are diverse range of biological activities and chemical structure which are used by bio pharmaceutical companies. Actually, seaweeds are an important source of carbohydrate, polyphenols, sterols, terpenoids, protein enzymes, fiber, lipids, vitamins including: A,B1, B2, B6, niacin and C, and are rich in iodine, potassium, iron, magnesium and calcium.
\end{abstract}

Keywords: Fucoidan, Fucoxanthin, Marine algae, Phloroglucinol, Phlorotannin, Seaweed, Sterol, Terpenoid

\section{Introduction}

Marine algae are simple organisms with diverse group, consisting of unicellular $(3-10 \mu \mathrm{m})$ to multicellular forms (kelps up to 70 metres). In the marine environment, they play an important role as a source of food and forming habitats. As a largest primary producer in the marine environment, they support life in the marine ecosystem through the production of oxygen, by building food webs, and by providing habitats for different organisms. More than $90 \%$ of global photosynthesis is contributed by algae and in the marine ecosystem algae are one of the leading primary producers. They consist of diverse group of large macro and micro algae. Microalgae are distributed throughout the ocean. Macroalgae mainly grow in the littoral zone, where they are constantly exposed to diverse fluctuating environmental conditions like oxidative stress, temperature and salinity which make them have great adaptations [1]. Seaweeds are considered very attractive sources for the screening of biologically active compounds, due to their huge biodiversity and safety. They synthesize numerous valuable antioxidants including carotenoids, tocopherol, ascorbic acid, chlorophyll derivatives, terpenoids, polysaccharides, phlorotannins, polyphenols and mycosporine-like amino acids. Marine algae are also a significant source of minerals, vitamins, proteins, fibres and poly unsaturated fatty acids. The beneficial effects upon the consumption of marine algae have also been demonstrated in different investigations. Various dietary antioxidants have shown remarkable promise as effective agents for chronic disease prevention and treatment by reducing oxidative stress, which has been involved in the development of many chronic diseases including cancer. Therefore, modification in dietary habits for reducing the incidence of these diseases, especially by increasing consumption of functional foods rich in antioxidants are increasingly supported. An algal antioxidant-mediated mechanism enhances the host's defence by increasing activation of nonspecific immune system were hypothesized as a contributing factors in the inhibition of many diseases. Chemical composition of edible seaweeds varies with species, habitats, maturity and environmental conditions like climate [2].

\section{Polyphenolic metabolites}

Polyphenols are extensively studied class of marine 
algal metabolites for their distinctive antioxidant, antiinflammatory activities, and effectiveness for the management of cardiovascular diseases, diabetes, hypertension, neurodegenerative diseases, and cancer [3]. Brown algae are a rich source of phenolic metabolites with a wide structural diversity. Phenolics refer to monomeric, oligomeric or polymeric compounds with an aromatic ring bearing one or more hydroxyl substituents, and include phenolic acids, flavonoids, bromophenols, and phlorotannins [4].

\section{Phlorotannins}

Phlorotannins are among the widely exploited bioactive polyphenols and have a wide range of molecular sizes $(400-400,000 \mathrm{Da})$ and can occur in variable concentrations $(0.5-20 \% \mathrm{dw})$ in brown algae [4]. Phlorotannins are stored in special vesicles (physodes) and are presumed to be the defense compounds involved in protection against stress conditions. Based on the monomers linkage, phlorotannins can be classified into four subclasses: fucols (phenyl linkage), fucophlorethols (ether and phenyl linkage), fuhalols and phlorethols (ether linkage) and carmalols and eckols (dibenzodioxin linkage) [5]. Antimicrobial activity of phlorotannins against food-borne bacteria and algae have been reported [4]. Phlorotannins are effective inhibitors of tyrosinase and melanin synthesis and absence of cytotoxic effects has been confirmed. Anti- inflammatory properties, antioxidant, antiphotocarcinogenic effect, antihypertension, therapeutic potential in arthritis treatment, protection against vascular diseases and protection against glucose-induced oxidative stress have also been reported [4]. A concise report about the antidiabetic effects of phlorotannins and their different mechanisms of action are reviewed. Accordingly, the majority of phlorotannins have indicated $\alpha$ - amylase and $\alpha$-glucosidase inhibitory effects, increase glucose uptake in skeletal muscles, inhibition of PTP1B, and improving insulin sensitivity [3].

\section{Phloroglucinol}

Phloroglucinol is a polyphenolic compound that chemical structure includes an aromatic phenyl ring with three hydroxyl groups. Like other phenolic compounds, phloroglucinol shows a variety of biological activities such as antioxidant, antiinflammatory, antidiabetic antimicrobial, antiallergic, and anti-HIV, by which has attracted attention for the development of new drugs. The eventual toxicity of phloroglucinol in normal cells has not been reported yet. Rather, it has been shown cytoprotective effects on oxidative stress induced cell damage in several models. Dryofragin, a phloroglucinol derivative, isolated from Dryopteris fragrans (L.) Schott exhibited potential anticancer activity in human breast cancer cells [5].

\section{Bromophenols}

Bromophenols (brominated phenolic derivatives) are famous for their antidiabetic activities. These activities are primarily attributed to the inhibition of $\alpha$ glucosidase and Protein tyrosine phosphatase 1B (PTP1B). Marine bromophenols have been mainly isolated from red algae belongs to the family Rhodomelaceae. They typically consist of monomeric and dimeric units of brominated 3,4-dihydroxybenzyl alcohols and alkyl ethe. increased bromination by chemical modification would increase the potency of PTP1B inhibition in bromophenols. The development of safe and selective antidiabetic drugs from bromophenols remains a challenging task because of their low availability in algae. Also, many studies are in need to confirm their bioavailability, safety, and side effects before therapeutic applications. HPN (3,4Dibromo-5-(2-bromo-3,4-dihydroxy-6

(isopropoxymethyl) benzyl)benzene-1,2-diol) is a synthetic derivative of a natural bromophenol found from the red alga R. confervoides. HPN is currently undergoing extensive investigations for its therapeutic potential as an antidiabetic agent. Other than the antidiabetic properties, some synthetic bromophenol derivatives prepared by acylation of benzoic acids with substituted benzenes are reported of possessing weak Angiotensin-converting enzyme (ACE) inhibitory activities [3].

\section{Polysaccharides}

Polysaccharides are polymers of monosaccharides linked by glycosidic bonds resulting in complex molecular structures. Large amounts of polysaccharides are present in the seaweed cell walls conferring flexibility and strength. It composition differs according to several intrinsic and extrinsic factors, namely seaweed specie, age and season [4]. Class Rhodophyceae (red algae) contains cellulose, porphyran, xylans, floridean starch, mannans and sulfated galactans such as carrageenans and agars. Polysaccharides in Chlorophyceae (green algae) are cellulose, sulfated galactans, ulvan, starch, xylans and $\beta$ D-mannans. Phaeophyceae (brown algae) is rich in anionic polysaccharides such as alginate, fucoidan (sulfated fucose rich glycans) and neutral polysaccharides including cellulose, mannitol and laminarin [3]. The interest in polysaccharides have been increase due to their biological activities such as anticancer and immunomodulatory effects that are structural-dependent. The benefit of algal polysaccharides is partly due to the high contents of indigestible dietary fibers. These fibers increase the 
feelings of satiety and aid in the digestive process through their bulking capacity. Consumption of seaweed fiber could influence to reduce chronic diseases, such as obesity, hypertension and diabetes [3].

\section{Alginates}

Alginates which comprise nearly up to $40 \%$ of the dry weight of algae are well-known for their anti- obesity effects. Alginates are linear unbranched polysaccharides with $\alpha$-l-guluronate and $\beta$-d mannuronate residues. They are hydrophilic, anionic and renowned for their ability to form hydrogels that have many applications in biomaterial research and pharmaceutical and food industry. Alginate consumption can delay gastric clearance, stimulate stretch receptors in the gut and attenuate nutrient absorption, thereby increasing the feeling of satiety and reduce energy intake. A further interesting aspect of alginate is that it could affect modulating the intestinal microflora population, which could positively affect to reduce the weight gain [3].

\section{Fucoidans}

Fucoidan consist mostly of sulfated L-fucose and small propor-tionsof glucose, xylose, galactose, mannose and anduronic acids. Chemical structure of this compound is complex and different structures appears depending on the seaweed specie from which it is isolated. Several biological activities including antioxidant, antiangiogenic, antiproliferative, antiviral, antiinflammatory, anticoagulant, antilipidemic and immunomodulatory have been, recently, attributed to the fucoidans. Nowadays, some applications have been emerged and fucoidan is used as additive to cosmetics, drinks and health foods. Fucoidan isolated from Cladosiphon okamuranus inhibited the proliferation of MCF-7 cells in a time and dose dependent manner, and induced apoptosis through a caspase-8-dependent pathway, without any effect on the viability of normal human mammary epithelial cells [4]. Xue et al., (2013), showed that intraperitoneal injection of fucoidan extract in 4T1 xenograft female Balb/c mice, inhibited tumor growth, angiogenesis and suppressed lung metastasis of breast cancer [6].

\section{Terpenoids}

Terpenes are lipophilic secondary metabolites made up of isoprene units, and can be grouped into hemi-, mono-, sesqui-, di-, sester-, tri-, and tetraterpenoids (carotenoids). Some diterpenes exclusive to marine organisms have been suggested as taxonomic markers [5].

\section{Carotenoids}

Carotenoids are tetraterpenoids with a specific linear C40 molecular backbone and are represented by more than 600 known natural structural variants. They are colorful pigments synthesized in plants, seaweeds and other photosynthetic organisms as well in some non photosynthetic bacteria and are involved in photosynthesis, photomorphogenesis, photoprotection and hormonal synthesis. Carotenoids are usually divided in two general classes: xanthophylls (which have at least one oxygen atom) and carotenes (containing only carbon and hydrogen atoms). Fucoxanthin, violaxanthin and lutein belong to the xanthophylls class, while $\beta$-carotene is the most common carotene. In seaweed, carotenoids profiles are used to classify seaweed into brown, red and green. The structure of carotenoids strongly affects their activity (e.g., presence of functional groups in the terminal rings increase the antioxidant potential). The antioxidant activity of carotenoids is mainly due to its ability to quench singlet oxygen and scavenging free radicals. Their antioxidant property is one of the main mechanisms by which they prevent oxidative stressrelated diseases, namely cancer, cardiovascular and neurodegenerative diseases. In vitro and in vivo studies have shown that carotenoids may protect against several types of cancer. These studies are consistent with data from epidemiological studies that showed an inverse correlation between consumption of a carotenoids rich diet and the risk of developing cancer [4].

\section{Fucoxanthin}

Attending to the mechanisms of anticancer activity of fucoxanthin, its antioxidant properties have an important role in cancer chemoprevention. Beside the antioxidant activity, some studies have reported a prooxidant effect of fucoxanthin on cancer cells, leading to increases of ROS. This could be a possible strategy to induce cell death in cancer cells. However, other mechanisms have been described and are based on the regulatory effect of fucoxanthin on biomolecules related to cell cycle arrest and metastasis and cell death. Fucoxanthin exhibits antiproliferative potential in different types of carcinomas including breast cancer (MCF-7) cells. Apoptosis induction has been suggested one of the mechanisms by which fucoxanthin inhibit proliferation of cancer cells [4]. Rwigemera et al., (2014 and 2015) showed that fucoxanthin and its main metabolite, fucoxanthinol, inhibited the viability of MDA-MB-231 and MCF-7 cells with induction of apoptosis. In MDA-MB-231 cells, anticancer effect of fucoxanthin and fucoxanthinol seems to be mediated by inhibition of NF-kB pathway $[7,8]$. Although the anticancer activity 
of fucoxanthin is reasonably known for several types of cancers, in breast cancer the data available are very limited. Consequently, further investigations are needed to assess the details of the effect and the underlying molecular mechanisms of fucoxanthin against breast cancer cells

\section{Protein and peptides}

The protein content of marine algae varies greatly. Most seaweed proteins have a high nutritional value, being a rich source of glutamic acid, aspartic acid and leucine, whereas tryptophan, threonine, lysine, histidine, sulphur amino acids are limiting. The protein digestibility is limited by the nonprotein fraction, which accounts for $10-20 \%$ of the nitrogen content [5]. Peptides are renowned for their anti-hypertensive (ACE-inhibitory) effects. The majority of the algaederived ACE-inhibitory peptides consisted of 2-5 amino acids (AA). Many of them are competitive ACEinhibitors, which could either bind with the active site or inhibitor binding site of ACE altering its conformation. Tryptophan, tyrosine, proline or phenylalanine at the C-terminal and presence of a branched-chain aliphatic AA at the Nterminal has seen favorable for peptides to act as competitive ACEinhibitors [3].

\section{Lipids}

Seaweeds report containing a relatively low amount of fatty acids that represent up to $2 \%$ of its dry weight. Mono and polyunsaturated fatty acids (MUFA and PUFA) reported exhibiting ample therapeutic effects against T2DM in glucose-intolerant subjects by improving insulin sensitivity. The stimulation of hepatic antioxidants is related to the antidiabetic properties of these bioactives. One possible mode of action would be the up-regulation of glucose transporter type 1 (GLUT1) and type 4 (GLUT4) proteins in the cell membrane. The substitution of saturated fatty acids with monounsaturated fatty acids (MUFA) was found to improve insulin sensitivity in healthy and glucoseintolerant subjects. More than MUFA, PUFA receives increased market demand due to its desirable functionalities in upregulating physiological well-being by mediating cellular and tissue metabolism. PUFA includes omega-3 and omega-6 fatty acids. Macroalgae report containing lower levels of polyunsaturated fatty acids compared to microalgae, which is the reason for being used of microalgae in the functional food industry. A recent study describes tetradecanoic acid isolated from Sargassum wightii as a potential noncompetitive inhibitor of $\alpha$-Glucosidase and $\alpha$-Amylase. The binding energy of tetradecanoic acid with $\alpha$ Glucosidase and $\alpha$-Amylase has shown better affinity, comparably higher than commercial inhibitor, acarbose [3].

\section{Sterols}

Phytosterols structurally and functionally resemble cholesterol, differing at C-24 in having an alkyl substitution on the side chain. The side chain of phytosterols usually contains 9 or 10 carbon atoms as opposed to 8 carbon in cholesterol. The sterol nucleus, 1, 2-cyclopentanoperhydrophenanthrene, comprising of four fused rings (A, B, C, and D), attaches a $\hat{a}-$ hydroxyl group (3-OH) at $\mathrm{C}-3$ and a long flexible side chain at $\mathrm{C}-17$. The hydrophilic $3-\mathrm{OH}$ usually contributes to hydrogen bonding with other hydrophilic groups, while the conformation and length of the side chain and the stereochemistry of the C-24 alkyl group are key to intermolecular interactions. Like cholesterol, algal sterols can exist either in free form or conjugated with other molecules such as fatty acids (for example, oleate) and sugars (most often, glucose), through covalent linkage with $3-\mathrm{OH}$. The most abundant sterol in macroalgae is fucosterol, which differs from its close neighbors, stigmasterol, sitosterol, and campesterol, also at its hydrocarbon tail. The algae sterols can be noted saringasterol, isofucosterol, $\hat{a}-$ sitosterol, ergosterol, poriferasterol, cholesterol. Algae sterols has beneficial effects, including inhibition of antioxidant, anticancer, antiinflammatory, cholesterol absorption, antidiabetic, antibacterial and antifungicidal actions. [9].

\section{Conclusion}

The nutritional value of edible algae, together with its health beneficial bioactive substances, makes seaweeds a suitable functional food ingredient that would enhance physiological well-being. Interestingly, many of the algal metabolites, including polysaccharides, PUFAs, polyphenols, sterols, terpenoids and peptides, have demonstrated potential towards remedying many diseases, including cancer, diabetes, obesity, hypertension and etc. Hence, the consumption of algae would improve physiological well-being. With modern advances in biotechnology, extraction, identification, and evaluation of bioactive compounds from natural resources have become fast and easy. Studies on the interaction of these metabolites within cellular systems could provide useful information regarding molecular mechanisms of actions and parameters such as dose requirements, efficiency, and bioavailability. Further studies on the structure-activity relationship would widen the research perspectives and provide insights into synthesizing derivatives of algal natural products, which could be resourceful drug candidates for manufacturing pharmaceuticals. 


\section{References}

1. Anbuchezhian R, Karuppiah V, Li Z. Prospect of marine algae for production of industrially important chemicals. Algal biorefinery: Integrat Approach. 2015; 195-217.

2. Namvar F, Baharara J, Mahdi AA. Antioxidant and anticancer activities of selected Persian Gulf algae. Indian J Clin Biochem. 2014; 29(1): 13-20.

3. Fernando IPS, Ryu B, Ahn G, Yeo IK, Jeon YJ. Therapeutic potential of algal natural products against metabolic syndrome: A review of recent developments. Trend Food Sci Technol. 2020; 97: 286-299.

4. Balboa EM, Conde E, Moure A, Falqué E, Domínguez $H$. In vitro antioxidant properties of crude extracts and compounds from brown algae. Food chem. 2013; 138(2-3): 1764-1785.

5. Pádua D, Rocha E, Gargiulo D, Ramos AA. Bioactive compounds from brown seaweeds: Phloroglucinol, fucoxanthin and fucoidan as promising therapeutic agents against breast cancer. Phytochem Lett. 2015; 14: 91-98.
6. Xue M, Ge Y, Zhang J, Liu Y, Wang Q, Hou L, Zheng Z. Fucoidan inhibited 4T1 mouse breast cancer cell growth in vivo and in vitro via downregulation of Wnt/ $\beta$-catenin signaling. Nutr Canc. 2013; 65(3): 460468.

7. Rwigemera A, Mamelona J, Martin LJ. Inhibitory effects of fucoxanthinol on the viability of human breast cancer cell lines MCF-7 and MDA-MB-231 are correlated with modulation of the NF-kappaB pathway. Cell Biol Toxicol. 2014; 30(3): 157-167.

8. Rwigemera A, Mamelona J, Martin LJ. Comparative effects between fucoxanthinol and its precursor fucoxanthin on viability and apoptosis of breast cancer cell lines MCF-7 and MDA-MB-231. Anticanc Res. 2015; 35(1): 207-219.

9. Hannan MA, Sohag AAM, Dash R, Haque MN, Mohibbullah M, Oktaviani DF, Hossain MT, Choi HJ, Moon IS. Phytosterols of marine algae: Insights into the potential health benefits and molecular pharmacology. Phytomed. 2020; 69: 153-201.

\section{ALKHAS}

Copyright: (C) 2021 The Author(s); This is an open-access article distributed under the terms of the Creative Commons Attribution License (http://creativecommons.org/licenses/by/4.0), which permits unrestricted use, distribution, and reproduction in any medium, provided the original work is properly cited.

Citation: Ghafarizadeh A, Niroomand A. Marine Algae as a New Source of Bioactive Compositionsin. ALKHAS. 2021; 3(4): 1-5.

https://doi.org/10.47176/alkhass.3.4.1 